\title{
Clinical application of specific antibody against glypican-3 for hepatocellular carcinoma diagnosis
}

\author{
YU JuanPing $^{1 \dagger}$, MA Qiang ${ }^{1 \dagger}$, ZHANG Bin $^{1}$, MA RuiJuan ${ }^{1}$, XU XiGuang ${ }^{2}$, \\ LI MingSong ${ }^{2}$, XU WeiWen ${ }^{1 *} \&$ LI Ming ${ }^{1 *}$ \\ ${ }^{1}$ Institute of Antibody Engineering, School of Biotechnology, Southern Medical University, Guangzhou 510515, China; \\ ${ }^{2}$ Department of Digestive Diseases, Nanfang Hospital, Southern Medical University, Guangzhou 510515, China
}

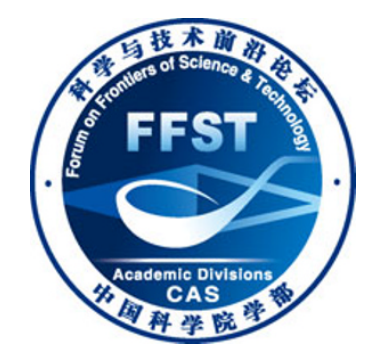

Received November 27, 2012; accepted January 10, 2013

\begin{abstract}
Glypican-3 (GPC3) is a promising tumor marker for hepatocellular carcinoma (HCC) diagnosis with high sensitivity and specificity. The aim of this study was to establish an immunohistochemical detection method for GPC3 using the 7D11 monoclonal antibody (7D11 mAb) and evaluate its application for HCC diagnosis. The feasibility of the 7D11 mAb was evaluated by immunohistochemistry performed on adjacent normal liver and intrahepatic cholangiocarcinoma (ICC) samples, Furthermore, the serum GPC3 levels were evaluated in 40 HCC patients, 7 ICC patients and 50 healthy donors. The results showed that GPC 3 was expressed in $85 \%$ of HCC tissues (34/40), but was undetectable in ICC tissues and adjacent normal tissues.GPC3 was significantly increased in the serum of HCC patients $(17 / 40,42.5 \%)$ but was undetectable in the serum of ICC patients $(0 / 7,0 \%)$ and healthy donors $(0 / 50,0 \%)$. This prospective study evaluated the clinical usefulness of 7D11 mAb for GPC3 detection in HCC patients. In conclusion, the use of 7D11 $\mathrm{mAb}$ might be good for GPC3 large-scale applications for clinical diagnosis of HCC.
\end{abstract}

glypican-3, 7D11 monoclonal antibody, hepatocellular carcinoma, immunohistochemistry

Citation: Yu J P, Ma Q, Zhang B, et al. Clinical application of specific antibody against glypican-3 for hepatocellular carcinoma diagnosis. Sci China Life Sci, 2013, 56: 234-239, doi: 10.1007/s11427-013-4451-y

Hepatocellular carcinoma (HCC), the fifth most common malignancy in humans, accounts for $6 \%$ of all cancers worldwide, with an estimated half million new cases diagnosed globally per-year [1]. It is the second most common malignancy in China [2], and currently, imaging techniques are important for the detection of HCC. However, the accuracy of imaging techniques such as computed tomography scanning, ultrasonography or magnetic resonance imaging is relatively insufficient for the diagnosis of small lesions, or dysplastic and cirrhotic nodules. Therefore, suitable biochemical markers to differentiate HCC from benign liver lesions are urgently needed.

Currently, $\alpha$-fetoprotein(AFP) is the most widely used

$\dagger$ Contributed equally to this work

*Corresponding author (email: mingli2006_2006@126.com; xu_sandy2006@126.com) tumor marker for the diagnosis and detection of HCC. However, AFP is significantly increased in the serum of patients with chronic liver disease, $15 \%-58 \%$ of patients with chronic hepatitis, liver repair after injury or cirrhosis, $11 \%-47 \%$ with cirrhosis [3].

Therefore, specific tumor markers are required for early detection of tumors and prognosis monitoring based on translational medicine. Glypican-3 (GPC3) is a member of the glypican family of heparan-sulfate proteoglycans, which are linked to the cell surface through a glycosyl phosphatidyl inositol anchor [4]. The expression levels of GPC3 are high in some embryonic tissues, such as the liver. However, it is absent in the corresponding normal adult tissue. Moreover, GPC3 is expressed in a large proportion of HCC patients $[5,6]$. Several studies indicated that GPC3 protein 
levels increased in the serum of patients with HCC, and was lower in the serum of healthy humans and patients with benign liver diseases [7-9]. These studies suggest that GPC3 is highly expressed in HCC patients, but not in adjacent normal liver or benign liver lesions [10-12]. However, there are no specific, economical GPC3 antibodies currently available with which to establish an immunohistochemical reagent to detect GPC3. This is a critical issue and an antibody specific for GPC3 is urgently needed.

In this study, we report on the preparation of an antihuman GPC3 mouse monoclonal antibody (mAb) 7D11 that can detect GPC3 in paraffin-embedded tissues through the optimization of antigen retrieval methods and concentration of the antibody. The results showed that 7D11 could detect GPC3 expression in both tumor tissues and serum with a high sensitivity and specificity.

\section{Materials and methods}

\subsection{Tissue and serum specimens}

Liver tissue samples were collected from patients undergoing hepatobiliary surgery in the South Hospital of Southern Medical University during the period from February 2011 to November 2011 and who did not receive radiotherapy or chemotherapy before surgery. HCC patients $(n=40)$ consisting of 11 well differentiated, 21 moderately differentiated, and 5 poorly differentiated tumors, and 3 cases without diagnosis were enrolled in the study. The age, sex, presence of cirrhosis, tumor differentiation, computed tomography scan analysis and hepatitis viral status were noted. From 21 cases samples of normal liver adjacent to HCC (normal liver) and 7 cases of ICC were collected. All of the specimens were formalin-fixed and embedded in paraffin, and tissue sections cut to a thickness of $3 \mu \mathrm{m}$ were placed on silanated slides and subjected to immunohistochemical analysis. Serum samples were also obtained from 40 patients with HCC, and 7 patients with ICC before the start of therapy. In addition, serum samples from 50 healthy donors were collected during the same period as the tissue samples. Approval was obtained from the regional ethics committee, and all subjects gave informed consent to participate.

\subsection{Immunohistochemical antibodies}

The 7D11 mAb was supplied by the Institute of Antibody Engineering, School of Biotechnology, Southern Medical University (Figure 1). 1G12 mAb was purchased from Biocare Medical (Catalog No. PM 396 AA). The secondary antibody working solution was purchased from Cell Marque (Lot: R1030-1742).

\subsection{Optimization of the experimental conditions}

We optimized the experimental conditions for immunofluo-
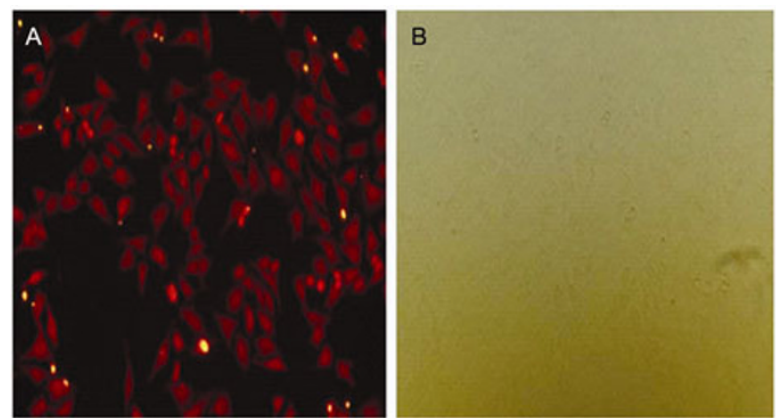

Figure 1 7D11 mAb identified by immunofluorescence (magnification 200x). Hepatoma cells (Huh7, Shanghai Cell Bank of the Chinese Academy of Sciences) that express GPC3 protein were used to test the specificity of 7D11 mAb by immunofluorescent staining. Figure 1A was observed using a fluorescent microscope. Figure $1 \mathrm{~B}$ was observed using a light microscope. Huh7 cells were recognized by 7D11 mAb and were observed as red fluorescence. This confirmed that 7D11 mAb could recognize GPC3 protein.

rescent staining using 7D11 mAb. The antigen retrieval treatment and the concentration of $7 \mathrm{D} 11 \mathrm{mAb}$ used were investigated.

We compared the use of citrate and EDTA buffer for pressure cooker heat-induced antigen retrieval, HCC tissue specimens were cut to $3 \mu \mathrm{m}$ thick sections, numbered 1-8, and deparaffinized with xylene, and rehydrated with a series of graded alcohols. Slices 1-4 were assigned to group A (citrate method) and slices 5-8 were assigned to group B (EDTA method). Citrate buffer $0.01 \mathrm{~mol} \mathrm{~L}^{-1} \mathrm{pH} 6.0$ (AR, Zhejiang, Changqing Chemical Industry Limited Company) was heated until boiling in an autoclave and the slices were placed into the boiling buffer and covered with a lid for 2 min. Sections were removed after cooling, and the slices were rinsed with distilled water, and washed with PBS. The same procedure was used for group B but using $1 \mathrm{mmol} \mathrm{L}^{-1}$ EDTA pH 8.0 (Ultra Pure, Sangon, CAS\#6381-92-6) instead of citrate. Next the sections were incubated with diluted monoclonal antibody (7D11) added dropwise at various concentrations: 1:400, 1:600, 1:800 and 1:1000. Detailed information is shown in Table 1 . The incubation was performed at room temperature for $30 \mathrm{~min}$, and sections were washed with PBS. The secondary antibody working solution was then incubated at room temperature for $30 \mathrm{~min}$.

\subsection{Immunohistochemical procedures}

Paraffin-embedded tissue sections were heated in a $60^{\circ} \mathrm{C}$ incubator for $20 \mathrm{~min}$, deparaffinized with xylene, and rehydrated with a series of graded alcohols. Antigen-retrieval was performed by heating the slides in $1 \mathrm{mmol} \mathrm{L}^{-1}$ EDTA buffer ( $\mathrm{pH}$ 8.0) in an autoclave. Sections were removed after cooling, rinsed with distilled water, washed with PBS, treated with horse serum albumin to block nonspecific staining, and immunostained using anti-GPC3 mAb (7D11and 1 G12) at a dilution of $1: 1000$. A slide using PBS 
Table 1 Optimization of the antigen retrieval methods and 7D11 mAb concentration

\begin{tabular}{|c|c|c|c|c|c|c|c|c|}
\hline \multirow{2}{*}{$\begin{array}{c}\text { Antigen retrieval methods } \\
\text { Tissue section No. }\end{array}$} & \multicolumn{4}{|c|}{$0.01 \mathrm{~mol} \mathrm{~L}^{-1}(\mathrm{pH} \mathrm{6.0)}$ citrate buffer } & \multicolumn{4}{|c|}{$1 \mathrm{mmol} \mathrm{L}^{-1}(\mathrm{pH} 8.0)$ EDTA buffer } \\
\hline & 1 & 2 & 3 & 4 & 5 & 6 & 7 & 8 \\
\hline 7D11 mAb concentration & $1: 400$ & $1: 600$ & $1: 800$ & $1: 1000$ & $1: 400$ & $1: 600$ & $1: 800$ & $1: 1000$ \\
\hline
\end{tabular}

instead of primary antibody was used as a negative control (Figure 2). Incubation was performed at room temperature for $30 \mathrm{~min}$ in a moisture chamber, and then washed with PBS. The secondary antibody working solution was added and sections incubated at room temperature for $30 \mathrm{~min}$. The slide sections were dipped in $10 \%$ hydrogen peroxide methanol for 10 min to block endogenous peroxidase activity, stained with diaminobenzidine hydrogen peroxide, and counterstained with hematoxylin for $3 \mathrm{~min}$.

\subsection{Immunohistochemistry}

Slides of 40 cases of HCC, 21 cases of normal liver and 7 cases of ICC tissue were prepared by cryosection, and stained with the 7D11 mAb according to the protocol described in Section 1.4, and stained with 1 G12 mAb according to the manufacturer's instructions. Staining using the two antibodies was then compared.

\subsection{Comparison of serum GPC3 and AFP levels}

To investigate the usefulness of a tumor marker for HCC, serum GPC3 and AFP levels from 40 cases of HCC, 7 cases of ICC and 50 cases of healthy individuals were measured. GPC3 and AFP were measured from the same serum samples. GPC3 was determined by 7D11 mAb with chemiluminescence analysis. AFP was measured using a commercially available time resolved fluoroimmunoassay kit (TRFIA) (Daan Gene Co., Ltd. of Sun Yat-sen University) according to the manufacturer's instructions.

\subsection{Data Analysis}

Statistical analysis of the results was performed using the
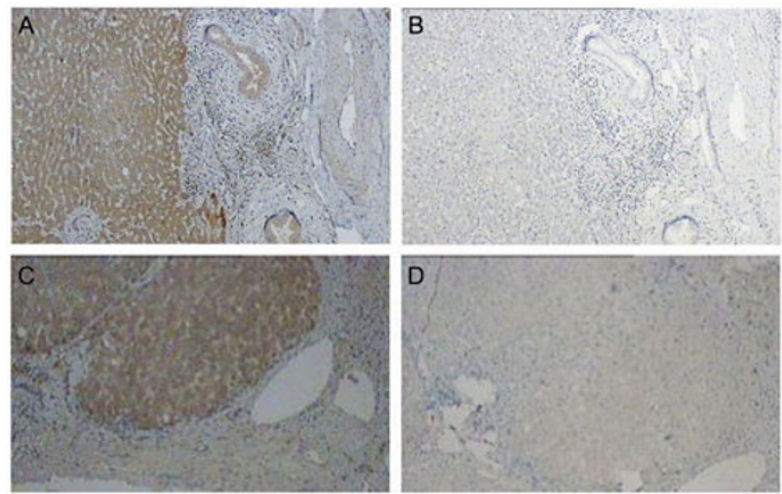

Figure 2 The negative and positive comparison. A, HCC, tissue stained with 1G12 mAb (magnification 200x). B, HCC, tissue treated with PBS (negative control)(magnification 200x). C, HCC, tissue stained with 7D11 $\mathrm{mAb}$ (magnification 200x). D, HCC, tissue treated with PBS (negative control)(magnification 200x). chi-square test, and differences were considered to be significant when $P<0.05$.

\subsection{Semi-quantification of immune staining}

The positive score range was as follows: $0-5 \%=0$, $6 \%-25 \%=1,26 \%-50 \%=2,51 \%-75 \%=3$, and $>76 \%=4$. Positive staining score: no coloring $=0$, slightly yellowish color $=$ 1 , brownish yellow $=2$, dark brown $=3$. The sum of the two scoring methods was as follows: $<2$ negative $(-), 2-3=$ weakly positive $(+), 4-5=$ positive $(++)$ and $6-7=$ strongly positive (+++).

\section{Results}

\subsection{Comparison of antigen retrieval methods}

When using the $0.01 \mathrm{~mol} \mathrm{~L}^{-1}$ citrate buffer ( $\mathrm{pH}$ 6.0) to reveal tissue antigens, positive staining was observed in cancer cells, and vascular endothelial cells around the cancer cells. Non-specific staining did not attenuate when the 7D11 $\mathrm{mAb}$ concentration was diluted (Figure $3 \mathrm{~A}$ and $\mathrm{B}$ ). When using $1 \mathrm{mmol} \mathrm{L}{ }^{-1}$ EDTA (pH 8.0), the 7D11 mAb dilution ratio was $1: 1000$, and positive staining of cancer cells was observed. Furthermore, anatomical structures and nuclear pulp were observed with better quality and degree of contrast, and without vascular endothelial and other non-specific staining (Figure 3C-E). A similar result was observed when using the $1 \mathrm{G} 12 \mathrm{mAb}$ (Figure $3 \mathrm{~F}-\mathrm{G}$ ). This suggested that use of $1 \mathrm{mmol} \mathrm{L}^{-1}$ EDTA ( $\mathrm{pH} 8.0$ ) was better than $0.01 \mathrm{~mol} \mathrm{~L}^{-1}$ citrate buffer ( $\mathrm{pH}$ 6.0). Therefore, the $1 \mathrm{mmol} \mathrm{L}^{-1}$ EDTA $(\mathrm{pH}$ 8.0) antigen retrieval method was used in this study using a dilution of 1:1000 for the 7D11 mAb.

\subsection{Immunohistochemistry of GPC3}

When 7D11 mAb and 1G12 mAb were used to assess GPC3 expression in different tissues, both antibodies displayed similar immunoreactivity (Figure 4). Table 2 summarizes the immunohistochemical results of GPC3 expression in $\mathrm{HCC}$, ICC and normal liver tissues. In the 7D11 mAb group, the positivity of GPC3 staining in HCC was $87.5 \%$ (35/40), and $0 \%$ in ICC and normal liver samples. When weakly positive samples were included as positive, these levels increased to $90 \%$ (36/40), $0 \%$ and $14.3 \%$ (3/21), respectively. In the $1 \mathrm{G} 12 \mathrm{mAb}$ group, the positivity in HCC, ICC, and normal livers were $82.5 \%, 0 \%$, and $0 \%$, respectively. When weakly positive samples were included as positive, these increased to $92.5 \%$ (37/40), $0 \%$ and $9.5 \%$ (2/21), respectively. 

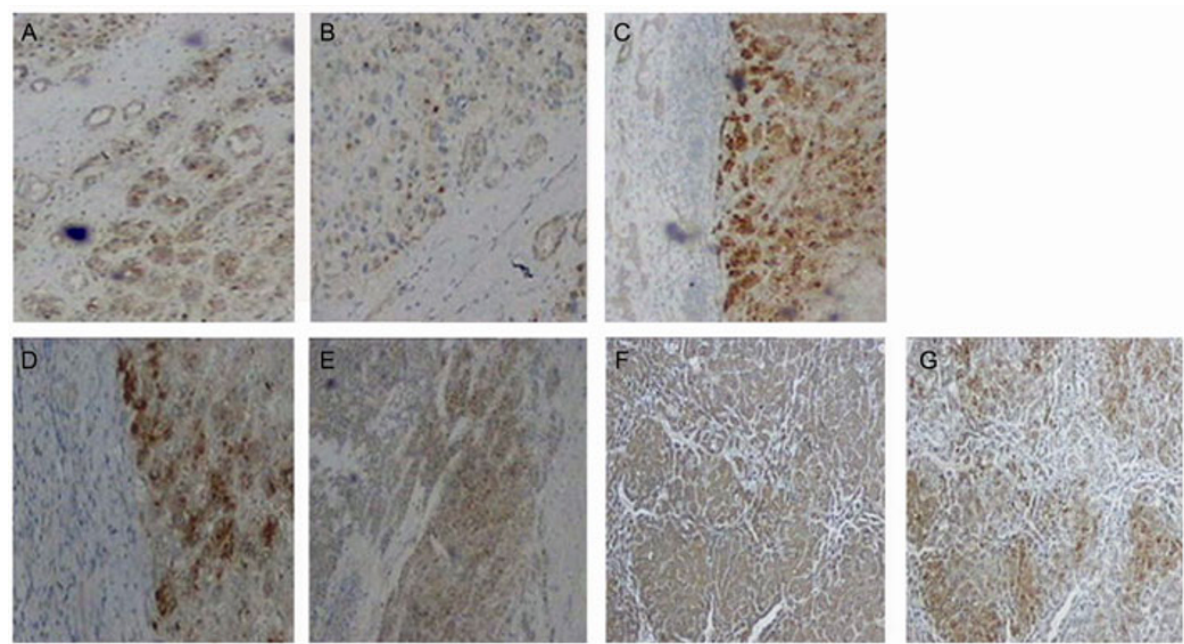

Figure 3 Comparison of different antigen retrieval methods and 7D11mAb dilution. A, Citrate buffer, 7D11mAb 1:400 (magnification 200x). B, Citrate buffer, 7D11 mAb 1:1000 (magnification 200x). C, EDTA, 7D11 mAb 1:600 (magnification 200x). D, EDTA, 7D11 mAb 1:1000 (magnification 200x). E, EDTA, 7D11 mAb 1:1000 (magnification 80x). F, Citrate buffer, 1G12 mAb 1:1000 (magnification 200x). G, EDTA, 1G12 mAb 1:1000 (magnification 200x).

Table 2 Glypican-3 expression in HCC, ICC and normal liver tissues

\begin{tabular}{|c|c|c|c|c|c|c|c|c|c|}
\hline & \multirow{2}{*}{ No. of specimens } & \multicolumn{4}{|c|}{$7 \mathrm{D} 11 \mathrm{mAb}$} & \multicolumn{4}{|c|}{$1 \mathrm{G} 12 \mathrm{mAb}$} \\
\hline & & +++ & ++ & + & - & +++ & ++ & + & - \\
\hline $\mathrm{HCC}$ & 40 & 18 & 17 & 1 & 4 & 17 & 16 & 4 & 3 \\
\hline ICC & 7 & 0 & 0 & 0 & 7 & 0 & 0 & 0 & 7 \\
\hline Normal liver & 21 & 0 & 0 & 3 & 18 & 0 & 0 & 2 & 19 \\
\hline
\end{tabular}
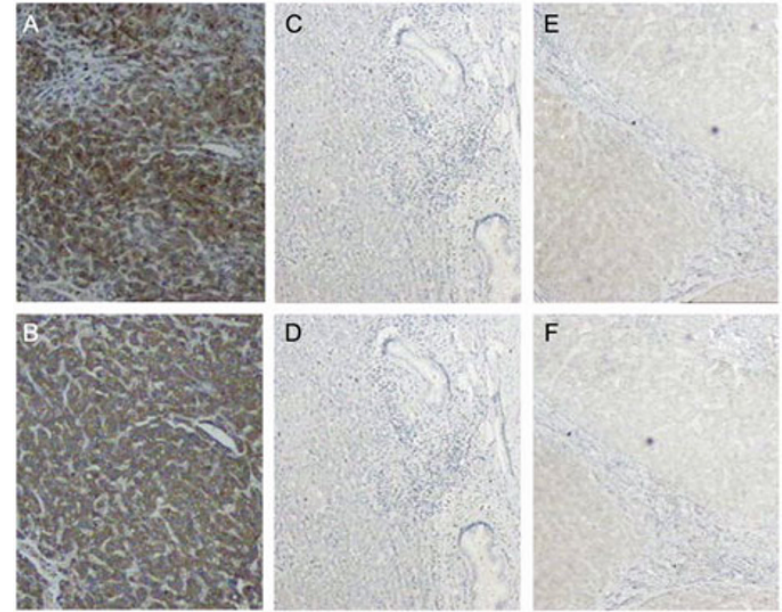

Figure 4 Staining comparison between GPC3 kit and commercial purchased 1 G12 kit. A, HCC tissue stained with 7D11 mAb (magnification 200×). B, HCC tissue stained with 1G12 mAb (magnification 200×). C, Normal tissue stained with 7D11 mAb (magnification $80 \times$ ) D, Normal tissue stained with $1 \mathrm{G} 12 \mathrm{mAb}$ (magnification $80 \times$ ). E, ICC tissue stained with 7D11 mAb (magnification 80x). F, ICC tissue stained with 1 G12 mAb (magnification $80 \times$ ).

\subsection{Serum Levels of GPC3 and AFP}

The results of immunohistochemistry showed that GPC3 is secreted in HCC tissue. Therefore, we investigated whether
GPC 3 could be detected in the serum of HCC patients using 7D11 mAb. A sandwich chemiluminescence immunoassay (CLIA) was used and the standard curve is shown in Figure 5. The results showed that serum GPC3 levels were significantly elevated in $45 \%$ (18/40) of HCC patients with values ranging from 32 to $545 \mathrm{ng} \mathrm{mL}^{-1}$, when a $30 \mathrm{ng} \mathrm{mL}^{-1}$ cutoff was used. GPC3 was undetectable in all ICCs and healthy donors (less than $3 \mathrm{ng} \mathrm{mL}^{-1}$ )(Table 3 ).

We also measured serum AFP levels in the same set of

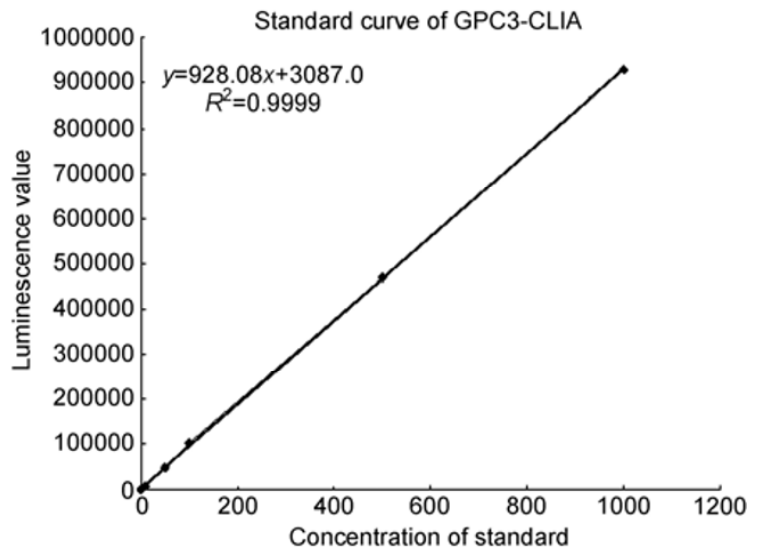

Figure 5 Standard curve of GPC3-CLIA. The standard was diluted to six concentrations, including $0,10,50,100,500$, and $1000 \mathrm{ng} \mathrm{mL}^{-1}$. The curve equation was $y=928.08 x+3087.0, r^{2}=0.9999$. 
samples. Serum AFP levels were elevated in 55\% (22/40) of HCC patients using a cutoff of $20 \mathrm{ng} \mathrm{mL}^{-1}$. AFP values were normal in healthy donors, and the values were elevated in $28.5 \%$ (2/7) of ICC patients (Table 3).

The tissue GPC3 positive rate was significantly higher than the serum GPC3 rate, but there was no difference in serum GPC3 concentration according to the degree of staining for GPC3 by immunohistochemistry in 40 cases of HCC. The positivity of serum GPC3 in HCC, ICC and healthy individuals was $45 \%(18 / 40), 0 \%(0 / 7)$ and $0 \%$ $(0 / 50)$, respectively. the serum AFP positive rate in HCC, ICC and healthy individuals was 55\% (22/40), 28.5\% (2/7) and $0 \%(0 / 50)$, respectively. Therefore, combining the serum GPC3 and AFP positive rates, the positive diagnostic rate of HCC was significantly increased $(29 / 40=72.5 \%)$.

\subsection{The relationship of GPC3 expression with tumor size, pathological grade, hepatitis and cirrhosis}

Specific relationships between GPC3 expression and tumor size, pathological grade, hepatitis and cirrhosis are shown in Table 4. The chi-square test was used for correlation analysis. The chi-square test showed no significant difference in the positive rate of GPC3 expression with these four aspects (tumor size $0.25>P<0.5$, pathological grade $0.975>P<0.99$, hepatitis $0.5>P<0.75$ and cirrhosis $0.1>P<0.25$ ).

\section{Discussion}

A confident diagnosis of cancer is a critical step prior to treatment. Early small HCC nodules are usually composed of well differentiated hepatocytes [13], so morphological criteria alone pose problems for differential diagnosis between high-grade dysplastic nodules and early HCC nodules, as one of the most reliable features of malignancy, can be absent. Thus, development of reliable tumor markers and reliable detection antibodies or technology is urgently needed [14].

GPC3 is a member of the glypican family of heparan-sulfate proteoglycans, which are linked to the cell surface through a glycosyl phosphatidyl inositol anchor [4]. GPC3 levels are significantly increased in a large proportion of HCC patients independent of AFP $[15,16]$. Therefore, GPC3 is a potential tumor marker for HCC. However, currently there is no economical available GPC3 detection reagent with high sensitivity, specificity for clinical applications. Our results show that the 7D11 mAb specifically recognized GPC3 protein (Figure 1) in HCC tissues (Figure 2 ). Results obtained with 7D11 mAb were similar to results with $1 \mathrm{G} 12 \mathrm{mAb}$ which is in common use.

To establish an immunohistochemical detection method, we optimized the antigen retrieval method and concentration of 7D11 mAb. During antigen retrieval treatment, 1 mmol L ${ }^{-1}$ EDTA buffer ( $\mathrm{pH}$ 8.0) provided better morphology than $0.01 \mathrm{~mol} \mathrm{~L}^{-1}$ citrate $(\mathrm{pH}$ 6.0), and when a concentration of 1:1000 of 7D11 mAb was used, immunohistochemistry results were better in terms of color and contrast and were without nonspecific staining. Immunohistochemistry results showed that GPC3 was expressed in $85 \%$ of HCC patients, but was undetectable in ICC and normal livers. This suggests that GPC3 could be a useful immunohistochemical marker for HCC diagnosis, and to distinguish HCC from benign liver disease and ICC. GPC 3 expression did not correlate with tumor size, or presence of hepatitis or cirrhosis. However, in patients with tumor size $<5 \mathrm{~cm}$ or hepatitis/cirrhosis GPC3-positive rates tended to be higher $(94.4 \%, 94.1 \%, 95 \%)$ than those who were GPC3 negative $(77.3 \%, 82.6 \%, 80 \%)$. Wang [17] reported that the expression of tissue GPC3 was 70\% (38/54) in HCC, $48 \%$ (14/29) in high-grade dysplastic or early HCC and $3 \%(2 / 65)$ in benign livers. 7D11 mAb has a high specificity and sensitivity for the detection of GPC3 by immunohistochemistry.

Serum GPC3 levels were also tested using the 7D11 $\mathrm{mAb}, \mathrm{GPC} 3$ was present in the serum of HCC patients $(45 \%, 18 / 40)$ when the cutoff was set at $30 \mu \mathrm{g} \mathrm{L} \mathrm{L}^{-1}$, and it

Table 3 Measurement of GPC3 (tissue), GPC3 (serum) and AFP (serum)

\begin{tabular}{|c|c|c|c|c|c|c|c|}
\hline \multirow{2}{*}{\multicolumn{2}{|c|}{ No. of specimens }} & \multicolumn{2}{|c|}{ Tissue of GPC3 } & \multicolumn{2}{|c|}{ Serum of GPC3 } & \multicolumn{2}{|c|}{ Serum of AFP } \\
\hline & & + & - & + & - & + & - \\
\hline $\mathrm{HCC}$ & 40 & 35 & 5 & 18 & 22 & 22 & 18 \\
\hline Healthy & 50 & - & - & 0 & 50 & 0 & 50 \\
\hline
\end{tabular}

Table 4 No correlation of GPC3 expression with tumor size, pathological grade, hepatitis and cirrhosis ${ }^{\text {a) }}$

\begin{tabular}{|c|c|c|c|c|c|c|c|c|c|}
\hline & \multicolumn{2}{|c|}{ Tumor size $(\mathrm{cm})$} & \multicolumn{3}{|c|}{ Pathological grading } & \multicolumn{2}{|c|}{ Viral hepatitis } & \multicolumn{2}{|c|}{ Cirrhosis } \\
\hline & $<5$ & $>5$ & $\mathrm{~W}$ & M & $\mathrm{P}$ & Yes & No & Yes & No \\
\hline Positive cases & 17 & 17 & 9 & 18 & 4 & 16 & 19 & 19 & 16 \\
\hline Negative cases & 1 & 5 & 2 & 3 & 1 & 1 & 4 & 1 & 4 \\
\hline Positive rate $(\%)$ & 94.4 & 77.3 & 81.8 & 85.7 & 80 & 94.1 & 82.6 & 95 & 80 \\
\hline
\end{tabular}

a) $\mathrm{W}$, well; $\mathrm{M}$, moderate; $\mathrm{P}$, poor. 
was undetectable in the serum of healthy donors $(0 \%, 0 / 50)$ and patients with ICC $(0 \%, 0 / 7)$. Serum AFP levels were elevated in $55 \%$ (22/40) of HCC patients and $28.5 \%$ (2/7) of ICC patients when the cutoff was set at $20 \mu \mathrm{g} \mathrm{L}^{-1}$ using the same serum samples. GPC3 was undetectable in healthy donors. In most cases of HCC, there was no significant correlation between serum GPC3 and AFP expression levels. However, the sensitivity of combination of GPC3 and AFP was $72.5 \%$ (29/40). Liu [18] also reported that serum GPC3 was $47.0 \%$ in HCC patients. Soha [19] reported that AFP (76.5\%) and GPC3 (63.5\%) were both increased in HCC patients, and that GPC3 levels were significantly higher in HCC patients than in cirrhotic patients and healthy donors. The pattern of these results was confirmed by our study.

In conclusion, the 7D11 mAb could be used to detect both serum and tissue GPC3 levels. Combined measurement of GPC3 and AFP could increase the sensitivity of detection compared with a single marker without compromising specificity.

This work was supported by the National High Technology Research and Development Program of China (2012AA020205), the Union Program of the Education Ministry, Guangdong Province (2011A090200028) and the Combination Project of the Education Ministry, Guangdong Province (2010B090400422).

1 Parkin D M, Bray F, Ferlay J, et al. Estimating the world cancer burden: Globocan 2000. Int J Cancer, 2001, 94: 153-156

2 Zhou L, Liu J, Luo F. Serum tumor markers for detection of hepatocellular carcinoma. World J Gastroenterol, 2006, 12: 1175-1181

3 Taketa K. $\alpha$-fetoprotein: revaluation in hepatology. Hepatology, 1990, 12: 1420-1432

4 Filmus J, Selleck S B. Glypicans: proteoglycans with a surprise. J Clin Invest, 2001, 108: 497-501

5 Zhu Z W, Friess H, Wang L, et al. Enhanced glypican-3 expression differentiates the majority of hepatocellular carcinomas from benign hepatic disorders. Gut, 2001, 48: 558-564

6 Zhou X P, Wang H Y, Yang G S, et al. Cloning and expression of MXR7 gene in human HCC tissue. World J Gastroenterol, 2000, 6: $57-60$

7 Hippo Y, Watanabe K, Watanabe A, et al. Identification of soluble NH2-terminal fragment of glypican-3 as a serological marker for early-stage hepatocellular carcinoma. Cancer Res, 2004, 64: 2418-2423

8 Zhang Q Y, Qin X, Zhen L, et al. Development of a competitive radioimmunoassay for glypican-3 and the clinical application in diagnosis of hepatocellular carcinoma. Clin Biochem, 2010, 43: 10031008

9 El-Shenawy S Z, Sabawi M M E1, Sheble N, et al. Diagnostic role of serum glypican-3 as a tumor marker for hepatocellular carcinoma. Nat Sci, 2012, 10: 32-38

10 Wang F H, Yip Y C, Zhang M, et al. Diagnostic utility of glypican-3 for hepatocellular carcinoma on liver needle biopsy. J Clin Pathol, 2010, 63: 599-603

11 Mariana C, Ian R W, Morris S, et al. Glypican-3 a novel serum and histochemical marker for hepatocellular carcinoma. Gastroenterology, 2003, 125: 89-97

12 Llovet J M, Chen Y, Wurmbach E, et al. A molecular signature to discriminate dysplastic nodules from early hepatocellular carcinoma in HCV cirrhosis. Gastroenterology, 2006, 131: 1758-1767

13 Roskams T, Kojiro M. Pathology of early hepatocellular carcinoma: conventional and molecular diagnosis. Semin Liver Dis, 2010, 30: 017-025

14 Bruix J, Morris S. Management of hepatocellular carcinoma: an update. Hepatology, 2011, 53: 1020-1022

15 Zhu Z W, Friess H, Wang L, et al. Enhanced glypican-3 expression differentiates the majority of hepatocellular carcinomas from benign hepatic disorders. Gut, 2001, 48: 558-564

16 Yutaka M, Shumpei I, Hiroko I, et al. Glypican-3, overexpressed in hepatocellular carcinoma, modulates FGF2 and BMP-7 signaling. Int J Cancer, 2003, 103: 455-465

17 Wang X Y, Degos F, Dubois S, et al. Glypican-3 expression in Hepatocellular tumors: diagnostic value for preneoplastic lesions and hepatocellular carcinomas. Hum Pathol, 2006, 37: 1435-1441

18 Liu H, Li P, Zhai Y, et al. Diagnostic value of glypican-3 in serum and liver for primary hepatocellular carcinoma. World J Gastroenterol, 2010, 16: 4410-4415

19 Soha ZE1 S, Maha ME1 S, Nashwa S, et al. Diagnostic role of serum glypican-3 as a tumor marker for hepatocellular carcinoma. Nat Sci, 2012, 10: 32-38

Open Access This article is distributed under the terms of the Creative Commons Attribution License which permits any use, distribution, and reproduction in any medium, provided the original author(s) and source are credited. 